\title{
Applying Differential Forms and the Generalized Sundman Transformations in Linearizing the Equation of Motion of a Free Particle in a Space of Constant Curvature
}

\author{
Joel M. Orverem ${ }^{1,2}$, Y. Haruna ${ }^{2}$, Bala M. Abdulhamid ${ }^{2}$ and Magaji Y. Adamu ${ }^{2}$ \\ ${ }^{1}$ Department of Mathematical Sciences, Federal University Dutsin-Ma, Katsina State, Nigeria \\ ${ }^{2}$ Department of Mathematical Sciences, Abubakar Tafawa Balewa University Bauchi, Bauchi State, Nigeria \\ Correspondence: Joel M. Orverem, Department of Mathematical Sciences, Federal University Dutsin-Ma, Katsina State, \\ Nigeria. E-mail: orveremjoel@yahoo.com
}

Received: June 23, $2021 \quad$ Accepted: July 21, $2021 \quad$ Online Published: August 25, 2021

doi:10.5539/jmr.v13n5p5 URL: https://doi.org/10.5539/jmr.v13n5p5

\begin{abstract}
:
Equation of motion of a free particle in a space of constant curvature applies to many fields, such as the fixed reduction of the second member of the Burgers classes, the study of fusion of pellets, equations of Yang-Baxter, the concept of univalent functions as well as spheres of gaseous stability to mention but a few. In this study, the authors want to examine the linearization of the said equation using both point and non-point transformation methods. As captured in the title, the methods under examination here are the differential forms (DF) and the generalized Sundman transformations (GST), which are point and non-point transformation methods respectively. The comparative analysis of the solutions obtained via the two linearizability methods is also taken into account.
\end{abstract}

Keywords: differential equations, Differential Forms, Equation of Motion, free particle, generalized Sundman transformation, linearization, point and non-point transformation, second order, space of constant curvature

\section{Introduction}

The equation of motion of a free particle in a space of constant curvature is a differential equation of second order. It finds applications in many areas as stated earlier. Such areas include spheres of gaseous stability, equations of Yang-Baxter, the study of fusion of pellets, the concept of univalent functions, and the static reduction of the second member of the Burgers classes, see (Nakpim \& Meleshko, 2010) and (Karasu \& Leach, 2009).

The equation

$$
y^{\prime \prime}+3 y y^{\prime}+y^{3}=0
$$

was mentioned in (Mahomed, 2007) in line with the invertible symmetry group transformation, and the point transformation

$$
X=\frac{1}{y}, Y=x+\frac{1}{y}
$$

was presented. The equation possesses the $\operatorname{sl}(3 \mathbb{R})$ algebra of Lie point symmetries (Karasu \& Leach, 2009). In other words, equation (1) admits the maximum eight-dimensional Lie algebra.

The method of differential forms which was first investigated by (Harrison, 2002), was used earlier in (Orverem, Tyokyaa \& Balami, 2017) and applied in (Orverem, Azuaba \& Balami, 2017) to investigate the possibility of linearizing equation (1). The GST method was first established in (Duarte, Moreira \& Santos, 1994) and later, in (Mustafa, Al-Dweik \& Mara'beh, 2013) where only the Laguerre form was investigated. Equation (1) was also stated in (Nakpim \& Meleshko, 2010) with a view of linearization through the generalized Sundman transformations (GST), but its solution was not obtained. This research is novel because the complete solution of equation (1) is obtained with the aid of the two methods under consideration DF and GST.

Note that (Nakpim \& Meleshko, 2010) presented the complete form of linearization through the GST. This method was also used by (Orverem, Haruna, Abdulhamid \& Adamu, 2021) to linearized the essential Emden differential equation as the complete solution was obtained.

In this present work, the authors apply the differential forms and the Sundman transformations methods in linearizing this 
equation of motion of a free particle in a space of constant curvature tagged as equation (1) above. Additionally, the comparison of the results of equation (1) from the two methods is also considered.

\section{Linearization of Equation (1) via the Differential Forms}

The starting point is a second-order ordinary differential equation

$$
y^{\prime \prime}=f\left(x, y, y^{\prime}\right)
$$

We assume a point transformation given by the variables

with a requirement that,

$$
X=F(x, y), \quad Y=G(x, y),
$$

$$
\frac{d^{2} Y}{d X^{2}}=0
$$

Using equation (4), we construct

$$
\frac{d Y}{d X}=\frac{G_{x}+G_{y} y^{\prime}}{F_{x}+F_{y} y^{\prime}}
$$

where $F_{x}+F_{y} y^{\prime} \neq 0$ and the subscripts $x$ and $y$ denote partial differentiation. The second derivative equation may be written in terms of a differential $d\left(\frac{d Y}{d X}\right)=0$ as

$$
\left(F_{x}+F_{y} y^{\prime}\right)\left(d G_{x}+y^{\prime} d G_{y}+G_{y} d y^{\prime}\right)-\left(G_{x}+G_{y} y^{\prime}\right)\left(d F_{x}+y^{\prime} d F_{y}+F_{y} d y^{\prime}\right)=0
$$

One can expand equation (6) and present it by collecting the like terms separately, as

$$
\begin{gathered}
F_{x} d G_{x}-G_{x} d F_{x}, \\
\left(F_{x} d G_{y}-G_{x} d F_{y}\right) y^{\prime}, \\
\left(F_{x} G_{y}-G_{x} F_{y}\right) d y^{\prime}, \\
\left(F_{y} d G_{x}-G_{y} d F_{x}\right) y^{\prime}, \\
\left(F_{y} d G_{y}-G_{y} d F_{y}\right) y^{\prime 2}, \\
\left(F_{y} G_{y}-G_{y} F_{y}\right) y^{\prime} d y^{\prime}=0 .
\end{gathered}
$$

Hence, one can write the above as

$$
T d y^{\prime}+\rho y^{\prime 2}+(\lambda+\delta) y^{\prime}+\sigma=0
$$

where

and have the 1 -forms

$$
T=F_{x} G_{y}-F_{y} G_{x}
$$

$$
\left.\begin{array}{l}
\rho=F_{y} d G_{y}-G_{y} d F_{y}, \lambda=F_{y} d G_{x}-G_{y} d F_{x}, \\
\sigma=F_{x} d G_{x}-G_{x} d F_{x}, \delta=F_{x} d G_{y}-G_{x} d F_{y} .
\end{array}\right\}
$$

We can rewrite equation (7) as

$$
d y^{\prime}=\alpha+\beta y^{\prime}+\gamma y^{\prime 2}
$$

where

$$
\alpha=\frac{-\sigma}{T}, \beta=\frac{-(\lambda+\delta)}{T}, \gamma=\frac{-\rho}{T} .
$$

Integrability of equation (10) demands that $d d y^{\prime}=0$, so that

$$
d \alpha+\left(\alpha+\beta y^{\prime}+\gamma y^{\prime 2}\right) \wedge \beta+y^{\prime} d \beta+2 y^{\prime}\left(\alpha+\beta y^{\prime}+\gamma y^{\prime 2}\right) \wedge \gamma+y^{\prime 2} d \gamma=0
$$

The $y^{\prime 3}$ term in equation (12) vanishes because $\gamma \wedge \gamma=0$, we expand and equate the coefficients of the other powers of $y^{\prime}$ to zero to have

$$
d \alpha=\beta \wedge \alpha, d \beta=2 \gamma \wedge \alpha, d r=\gamma \wedge \beta .
$$

We revisit equations (9), expand the differential, and obtain 
which can simply be presented as

$$
\begin{gathered}
\rho=F_{y}\left(G_{x y} d x+G_{y y} d y\right)-G_{y}\left(F_{x y} d x+F_{y y} d y\right), \\
\lambda=F_{y}\left(G_{x x} d x+G_{x y} d y-G_{y}\left(F_{x x} d x+F_{x y} d y\right),\right. \\
\sigma=F_{x}\left(G_{x x} d x+G_{x y} d y\right)-G_{x}\left(F_{x x} d x+F_{x y} d y\right), \\
\delta=F_{x}\left(G_{x y} d x+G_{y y} d y\right)-G_{x}\left(F_{x y} d x+F_{y y} d y\right),
\end{gathered}
$$

where

$$
\rho=A d x+B d y, \lambda=C d x+A d y, \sigma=D d x+E d y, \delta=E d x+H d y,
$$

Now,

$$
\begin{aligned}
& A=F_{y} G_{x y}-G_{y} F_{x y}, B=F_{y} G_{y y}-G_{y} F_{y y}, C=F_{y} G_{x x}-G_{y} F_{x x}, \\
& D=F_{x} G_{x x}-G_{x} F_{x x}, E=F_{x} G_{x y}-G_{x} F_{x y}, H=F_{x} G_{y y}-G_{x} F_{y y} .
\end{aligned}
$$

$$
\alpha=\frac{-(D d x+E d y)}{T}, \beta=\frac{-(C d x+E d x+A d y+H d y)}{T}, \gamma=\frac{-(A d x+B d y)}{T} .
$$

Putting $\alpha, \beta$, and $\gamma$ into equation (10) and dividing by $d x$ to convert the differential forms to functions, we have

$$
y^{\prime \prime}+f_{0}+f_{1} y^{\prime}+f_{2} y^{\prime 2}+f_{3} y^{\prime 3}=0,
$$

where the $f_{k}$ are given by

$$
f_{0}=\frac{D}{T}, f_{1}=\frac{(C+2 E)}{T}, f_{2}=\frac{(H+2 A)}{T}, f_{3}=\frac{B}{T} .
$$

Thus, it is necessary through the differential forms' procedure that equation (3) should be cubic in the first derivative obtained as equation (16).

Now, we define $K$ and $L$ as

$$
K=\frac{E}{T}, L=\frac{A}{T}
$$

and replace $D, C, H$ and $B$ in the 1-forms in equation (15) in favour of the $f_{k}, K$ and $L$, obtaining

$$
\alpha=-f_{0} d x-K d y, \beta=\left(K-f_{1}\right) d x+\left(L-f_{2}\right) d y, \gamma=-L d x-f_{3} d y .
$$

We also note that

$$
\frac{d T}{T}=\left(3 K-f_{1}\right) d x+\left(f_{2}-3 L\right) d y
$$

We see that the 1 -forms $\alpha, \beta, \gamma$ in equation (19) and $\frac{d T}{T}$ in equation (20) are now expressed in terms of these four known functions $K$ and $L$. The first three of these 1-forms can now be substituted into equation (13) on the various functions. If we do that, the first equation for $d \alpha$, gives the equation

$$
f_{0 y}-K_{x}=-K\left(K-f_{1}\right)+f_{0}\left(L-f_{2}\right)
$$

which is nonlinear in $\mathrm{K}$. The other equations give the results:

$$
-K_{y}+f_{1 y}+L_{x}-f_{2 x}=2 K L-f_{0} f_{3}
$$

and

$$
L_{y}-f_{3 x}=-L\left(L-f_{2}\right)+f_{3}\left(K-f_{1}\right)
$$

which are also nonlinear. However, we can simplify the situation by defining new variables:

$$
T=\frac{1}{W^{3}}, E=\frac{U}{W^{4}}, \quad A=\frac{V}{W^{4}},
$$

so that from equation (18)

$$
K=\frac{U}{W}, L=\frac{V}{W^{\prime}}
$$


and from equation (20)

$$
3 \frac{d W}{W}=\left(f_{1}-3 K\right) d x+\left(3 L-f_{2}\right) d y .
$$

The $d W$ equation (26) gives expressions for $W_{x}$ and $W_{y}$. The equation (21) after substitution for $W_{x}$, gives an expression

$$
U_{x}=W f_{0 y}-\frac{2}{3} U f_{1}-V f_{0}+W f_{0} f_{2}
$$

which is linear in $U, V$ and $W$. The equation (23) gives an expression

$$
V_{y}=W f_{3 x}+\frac{2}{3} V f_{2}+U f_{3}-W f_{1} f_{3}
$$

which is also linear. The equation (22) gives a linear expression

$$
V_{x}-U_{y}=\frac{U}{3} f_{2}+\frac{V}{3} f_{1}-W f_{1 y}+W f_{2 x}-2 f_{0} f_{3} W .
$$

The integrability condition on equation (26) gives a linear expression

$$
V_{x}+U_{y}=\frac{U}{3} f_{2}+\frac{V}{3} f_{1}+\frac{W}{3} f_{2 x}+\frac{W}{3} f_{1 y} .
$$

Equations (29) and (30) can be solved for $V_{x}$ and $U_{y}$. Thus we have expressions for all derivatives of $U, V$, and $W$, all of which are linear and homogeneous in the same variables. That is

$$
\begin{gathered}
d U=\frac{1}{3}\left(-2 U f_{1}-3 V f_{0}+W\left(3 f_{0 y}+3 f_{0} f_{2}\right)\right) d x+\frac{1}{3}\left(-U f_{2}+W\left(2 f_{1 y}-f_{2 x}+3 f_{0} f_{3}\right)\right) d y, \\
d V=\frac{1}{3}\left(V f_{1}+W\left(2 f_{2 x}-f_{1 y}-3 f_{0} f_{3}\right)\right) d x+\frac{1}{3}\left(3 U f_{3}+2 V f_{2}+W\left(3 f_{3 x}-3 f_{1} f_{3}\right)\right) d y, \\
d W=\frac{1}{3}\left(-3 U+W f_{1}\right) d x+\frac{1}{3}\left(3 V-W f_{2}\right) d y .
\end{gathered}
$$

We summarize all these relations in a fine matrix equation

$$
d r=M r
$$

where

$$
\begin{gathered}
r=\left(\begin{array}{c}
U \\
V \\
W
\end{array}\right) \text { and } M=P d x+Q d y, \\
P=\left(\frac{1}{3}\right)\left(\begin{array}{ccc}
-2 f_{1} & -3 f_{0} & 3 f_{0 y}+3 f_{0} f_{2} \\
0 & f_{1} & 2 f_{2 x}-f_{1 y}-3 f_{0} f_{3} \\
-3 & 0 & f_{1}
\end{array}\right) \\
Q=\left(\frac{1}{3}\right)\left(\begin{array}{ccc}
-f_{2} & 0 & 2 f_{1 y}-f_{2 x}+3 f_{0} f_{3} \\
3 f_{3} & 2 f_{2} & 3 f_{3 x}-3 f_{1} f_{3} \\
0 & 3 & -f_{2}
\end{array}\right) .
\end{gathered}
$$

For integrability of equation (34), $d d r=0$ giving

$$
d M=M \wedge M
$$

which is not zero since $M$ is a matrix. Substitution for $M$ in terms of $P$ and $Q$ gives the condition

$$
Q_{x}-P_{y}+Q P-P Q=0 .
$$

This matrix condition in equation (36) reduces to the following conditions:

$$
f_{0 y y}+f_{0}\left(f_{2 y}-2 f_{3 x}\right)+f_{2} f_{0 y}-f_{3} f_{0 x}+\left(\frac{1}{3}\right)\left(f_{2 x x}-2 f_{1 x y}+f_{1} f_{2 x}-2 f_{1} f_{1 y}\right)=0,
$$


and

$$
f_{3 x x}+f_{3}\left(2 f_{0 y}-f_{1 x}\right)+f_{0} f_{3 y}-f_{1} f_{3 x}+\left(\frac{1}{3}\right)\left(f_{1 y y}-2 f_{2 x y}+2 f_{2} f_{2 x}-f_{2} f_{1 y}\right)=0 \text {. }
$$

To summarize, we note that the original differential equation is cubic in $y^{\prime}$ presented in equation (16) with the coefficients satisfying equations (37) and (38).

Now, we shall construct the point transformations properly. We will need $U, V$ and $W$ therefore we need to solve equations (34). Once the equations are solved, we produce $K$ and $L$ from equation (25). To find the $F(x, y)$ and $G(x, y)$ which we are seeking, we revisit equations (9) and solve for $d F_{x}, d F_{y}, d G_{x}$ and $d G_{x}$. Solution for $d F_{x}$ and $d F_{y}$ gives

$$
d F_{x}=\frac{\left(F_{y} \sigma-F_{x} \lambda\right)}{T}, \quad d F_{y}=\frac{\left(F_{y} \delta-F_{x} \rho\right)}{T} .
$$

Solution for $d G_{x}$ and $d G_{y}$, shows that they satisfy the same equation, so we will write only equations for the derivatives of $F$. We note that

$$
\delta+\lambda=-T \beta \text { and } \delta-\lambda=d T,
$$

so, we can solve these equations for $\delta$ and $\lambda$. We can also substitute for $\sigma$ and $\rho$ in terms of $\alpha$ and $\gamma$. We finally get

$$
d F_{x}=-F_{y} \alpha+F_{x} \frac{\left(\beta+\frac{d T}{T}\right)}{2}, \quad d F_{y}=F_{x} \gamma+F_{y} \frac{\left(-\beta+\frac{d T}{T}\right)}{2} \text {. }
$$

We substitute for $\alpha, \beta, \gamma$ and $d T / T$ from equations (19) and (20) respectively in terms of the expressions obtained above, to with $f_{k}, K$ and $L$.

We now have two equations that can be expressed in matrix form as

$$
d R=Z R, d S=Z S
$$

where

$$
Z=\left(\begin{array}{cc}
\left(2 K-f_{1}\right) d x-L d y & f_{0} d x+K d y \\
-L d x-f_{3} d y & K d x+\left(f_{2}-2 L\right) d y
\end{array}\right), \quad R=\left(\begin{array}{l}
F_{x} \\
F_{y}
\end{array}\right) \text { and } \quad S=\left(\begin{array}{l}
G_{x} \\
G_{y}
\end{array}\right) .
$$

This linear equation set can be solved for $R$. There will be two independent solutions, which can be taken as $R$ and $S$ as seen in equation (39). Integrability is guaranteed by setting $d d R=0$.

Finally, we can solve

$$
d F=\left(\begin{array}{ll}
d x & d y
\end{array}\right) R, d G=\left(\begin{array}{ll}
d x & d y
\end{array}\right) S,
$$

and the two independent solutions can be taken as $F$ and $G$.

Now, we see that equation (1) is in the form of equation (16) and its coefficients, $f_{0}=y^{3}, f_{1}=3 y, f_{2}=f_{3}=0$ satisfy the conditions (37) and (38) above.

Construction of the $3 \times 3$ matrix gives

$$
M=\left(\begin{array}{ccc}
-2 y d x & -y^{3} d x & 3 y^{2} d x+2 d y \\
0 & y d x & -d x \\
-d x & d y & y d x
\end{array}\right)
$$

so that

$$
d r=\left(\begin{array}{c}
-2 y U d x-y^{3} V d x+W\left(3 y^{2} d x+2 d y\right) \\
y V d x-W d x \\
-U d x+V d y+y W d x
\end{array}\right)
$$

and $r=\left(\begin{array}{c}U \\ V \\ W\end{array}\right)$. Putting $U=0, d U=0$, so that

$$
d V=(V y-W) d x, \quad d W=W y d x+V d y
$$


We now see that $W_{x}=W y$ and $W_{y}=V$ which on integration, we have that $W=e^{x y+a(y)}$, for some function $a(y)$. Also, $V=e^{a(y)+x y}\left(x+a^{\prime}(y)\right)$, and considering the special solution $a(y)=1$, we have that

$$
U=0, \quad V=x e^{x y+1}, \quad W=e^{x y+1} .
$$

From equation (25), one sees that $K=0$ and $L=x$.

We also notice that $Z=\left(\begin{array}{cc}-3 y d x-x d y & y^{3} d x \\ -x d x & -2 x d y\end{array}\right)$. Setting $R=\left(\begin{array}{l}b \\ c\end{array}\right)$, we see that

$$
d R=\left(\begin{array}{c}
-b(3 y d x+x d y)+c y^{3} d x \\
-b x d x-2 c x d y
\end{array}\right),
$$

so that

$$
d b=\left(-3 b y+c y^{3}\right) d x-b x d y
$$

and

$$
d c=-b x d x-2 c x d y
$$

One sees from equation (41) that $b_{y}=-b x$, so that

$$
b=k e^{-x y}
$$

where $k$ is a constant. Differentiation of equation (43) with respect to $y$ gives

$$
b_{y}=-k x e^{-x y} \text {. }
$$

We notice from equation (42) that, $c_{x}=-b x$, and on substitution of $b$ from equation (43), we see that

$$
c_{x}=-k x e^{-x y}
$$

which indicates that $b_{y}=c_{x}$.

One can integrate equation (45) using the integration by parts to obtain

$$
c=k x y^{-1} e^{-x y}+k y^{-2} e^{-x y}+g(y) .
$$

On differentiation of equation (46) with respect to $y$, one sees that

$$
c_{y}=\frac{-k x e^{-x y}}{y^{2}}(x y+1)-\frac{k e^{-x y}}{y^{3}}(x y+2)+g^{\prime}(y) .
$$

We also note from equation (42) that

$$
c_{y}=-2 c x
$$

Equating equations (47) and (48) and simplifying, we have

$$
g^{\prime}+2 x g=2 k y^{-3} e^{-x y}-k x^{2} y^{-1} e^{-x y},
$$

which can be solved with the use of integrating factor. Solving equation (49) and further simplification, one arrives at

where $m$ is another constant.

$$
g=-k y^{-2} e^{-x y}-k x y^{-1} e^{-x y}+m e^{-2 x y},
$$

On substation of equation (50) into equation (46), equation (46) reduces to

$$
c=m e^{-2 x y} \text {. }
$$

Summarily,

$$
b=F_{x}=k e^{-x y} \text { and } c=F_{y}=m e^{-2 x y} .
$$

Taking $F_{y}=m e^{-2 x y}$, one can integrate with respect to $y$ and obtain 


$$
F=\frac{-m e^{-2 x y}}{2 x}+h(x)
$$

Now, differentiating equation (52) with respect to $x$ we see that

$$
F_{x}=\frac{m y e^{-2 x y}}{x}+\frac{m}{2 x^{2}} e^{-2 x y}+h^{\prime}(x) .
$$

Now, equations (51) and (53) are similar. Therefore, one can simplify this situation to see that

$$
h^{\prime}(x)=k e^{-x y}-m x^{-1} y e^{-2 x y}-\frac{m}{2 x^{2}} e^{-2 x y} .
$$

We truncate the last term of equation (54) since it is also the coefficient of the constant $m$, and integrate the result by parts to have

$$
h(x)=\frac{-k}{y} e^{-x y}-m y \ln x e^{-2 x y} .
$$

Therefore, equation (52) becomes

$$
F+k e^{-x y}\left(\frac{1}{y}\right)+m e^{-2 x y}\left(\frac{1}{2 x}+y \ln x\right)=0 .
$$

Without loss of generality, we let $e^{-x y}=e^{-2 x y}=\ln x=1$, and interchanging the variables of constant $m$, we have

$$
X=\frac{1}{y}, \quad Y=\frac{1}{y}+x
$$

as the linearizing point transformation. The result in equation (56) is in line with the one presented in (Mahomed, 2007) above.

\section{Linearization of Equation (1) via the GST Method}

The transformation that is defined to be

$$
u(t)=F(x, y), d t=G(x, y) d x,\left(G F_{y}\right) \neq 0,
$$

is referred to as the GST. It is essential to note that, equation (57) is a non-point transformation.

Equation (3) must take the form

$$
y^{\prime \prime}+f_{2} y^{\prime 2}+f_{1} y^{\prime}+f_{0}=0,
$$

with the aid of equation (57) to be transformed into a linear ODE

$$
u^{\prime \prime}+\beta u^{\prime}+\alpha u=\gamma,
$$

for some functions $\alpha(t), \beta(t)$ and $\gamma(t)$.

Taking the case $f_{3} \neq 0$ and $f_{5} \neq 0$ into account, we have the conditions that are sufficient for equation (58) to be linearizable via equation (57) to be:

$$
\begin{gathered}
f_{0 x}=\frac{2 f_{0}\left(f_{3}-f_{1} f_{3}\right)}{f_{3}}, \\
f_{2 x x y}=-f_{2 x y} f_{1}-f_{3 x y}-2 f_{2 x}^{2}-2 f_{2 x} f_{3}-f_{3 y} f_{1}+\left(f_{3 y} f_{5}\right) f_{3}^{-1}, \\
f_{2 x x x}=-f_{3 x x}-f_{1 x} f_{2 x}-f_{1 x} f_{3}+f_{2 x} f_{1}^{2}+f_{1}^{2} f_{3}-2 f_{1} f_{5}+f_{3}^{-1} f_{5}\left(f_{3 x}+f_{5}\right),
\end{gathered}
$$

and

$$
\begin{gathered}
f_{3} f_{5}\left(6 f_{0 y} f_{2 x}+2 f_{2 x y} f_{0}+4 f_{2 x} f_{0} f_{2}+2 f_{3 y} f_{0}+4 f_{0} f_{2} f_{3}+f_{1} f_{5}\right)-f_{3}^{2}\left(6 f_{2 x}^{2} f_{0}+12 f_{2 x} f_{1} f_{3}-6 f_{0 y} f_{5}+\right. \\
\left.6 f_{0} f_{3}^{2}\right)-f_{4} f_{5}^{2}-2 f_{5}^{3}=0
\end{gathered}
$$


where $f_{3}=f_{1 y}-2 f_{2 x}, f_{4}=2 f_{0 y y}-2 f_{1 x y}+2 f_{0} f_{2 y}-f_{1 y} f_{1}+2 f_{0 y} f_{2}+2 f_{2 x x}$ and $f_{5}=f_{2 x x}+f_{2 x} f_{1}+f_{3 x}+f_{1} f_{3}$.

We can find the functions $F$ and $G$ by solving the equations that follow:

$$
\begin{gathered}
F_{x}=0, \\
F_{y y}=\frac{F_{y} G_{y}+f_{2} F_{y} G}{G}, \\
G_{x}=\frac{G\left(f_{2 x x}+f_{2 x} f_{1}+f_{3 x}\right)}{f_{3}}, \\
G_{y}=\frac{G f_{3}\left(f_{2 x}+f_{3}\right)}{f_{5}} .
\end{gathered}
$$

The $\alpha, \beta$ and $\gamma$ from equation (59) are examined from the equations

$$
\begin{gathered}
\alpha=\frac{G\left(f_{0 y}+f_{0} f_{2}\right)-G_{y} f_{0}}{G^{3}}, \\
\beta=\frac{G_{x}+G f_{1}}{G^{2}}, \\
\gamma=\frac{\alpha F G^{2}-F_{y} f_{0}}{G^{2}} .
\end{gathered}
$$

Again, from equation (1), one sees that it is in the form of (58) with the coefficients $f_{0}=y^{3}, f_{1}=3 y, f_{2}=0$. Hence, one has the expressions for $f_{3}, f_{4}$ and $f_{5}$ as defined earlier to be $f_{3}=3, f_{4}=3 y$ and $f_{5}=9 y$ respectively.

Testing for the linearizability conditions as stated from equations (60) to (63), one sees that (1) satisfies all the conditions, and is hence linearizable by the GST method. Equations (64) through (67) gives $F_{x}=0, F_{y y}=\frac{F_{y}}{y}, G_{x}=0$ and $G_{y}=\frac{G}{y}$ respectively. One can take the simplest of the solutions $F=y^{2}, G=y$ so that $u=y^{2}, d t=y d x$.

Now, considering equations (68) to (70), one obtains $\alpha=2, \beta=3$, and $\gamma=0$ respectively. Therefore, equation (59) becomes

$$
u^{\prime \prime}+3 u^{\prime}+2 u=0 .
$$

Equation (71) is now a linear differential equation with the general solution

$$
u=c_{1} e^{-t}+c_{2} e^{-2 t},
$$

where $c_{1}, c_{2}$ are arbitrary constants. From (57), we now have that

$$
y=\left(c_{1} e^{-t}+c_{2} e^{-2 t}\right)^{1 / 2} .
$$

But $\frac{d t}{d x}=y$ that is

$$
d t=\left(c_{1} e^{-t}+c_{2} e^{-2 t}\right)^{1 / 2} d x,
$$

so that $t=x\left(c_{1} e^{-t}+c_{2} e^{-2 t}\right)^{1 / 2}=\phi(x)$, and finally,

$$
y=\left(c_{1} e^{-\phi(x)}+c_{2} e^{-2 \phi(x)}\right)^{1 / 2} .
$$

\section{Discussion and Conclusion}

It is important to know the genesis (origin) of the differential equation stated in (1). Considering the ODE

$$
y^{\prime \prime}+\lambda_{1} y^{k_{1}}+\lambda_{2} y^{k_{2}} y^{\prime}+\lambda_{3} y^{k_{3}} y^{\prime 2}=0,
$$

where $\lambda_{1}, \lambda_{2}, \lambda_{3}$ and $k_{1}, k_{2}, k_{3}$ are constants. If $\lambda_{3}=0$, equation (76) becomes

$$
y^{\prime \prime}+\lambda_{1} y^{k_{1}}+\lambda_{2} y^{k_{2}} y^{\prime}=0,
$$


where $\lambda_{1} \neq 0, \lambda_{2} \neq 0$. If $\lambda_{1}=1, \lambda_{2}=3, k_{1}=3$ and $k_{2}=1$, (77) is reduced to

$$
y^{\prime \prime}+y^{3}+3 y y^{\prime}=0
$$

which is similar to equation (1) stated earlier.

Using the transformation $Y=X a+b$, where $a$ and $b$ are constants, we see from equation (56) that $y=\frac{1-a}{b-x}$ or simply put,

$$
y=\frac{c}{b-x}
$$

where $c=1-a$.

Comparing the two results in (56) and (75), one sees that the method of differential forms gives the linearizing point transformation (56) which on another transformation yields the solution (79), while the GST method readily yields the solution as seen in (75). Both of the solutions are correct in their respect, but the solution (75) is more convincingly, the general solution of the equation given in (1).

Researchers can also look out for other approaches possible, suitable for the solution of this very important equation. This will help broaden the study of equation (1) considered in this research.

\section{References}

Duarte, L. G. S., Moreira, I. C., \& Santos, F. C. (1994). Linearization under nonpoint transformations. Journal of Physics A: Mathematical and General, 27(19), 739-743. https://doi.org/10.1088/0305-4470/27/19/004

Harrison, B. K. (2002). An Old Problem Newly Treated with Differential Forms : When and How Can the Equation y = $\mathrm{f}(\mathrm{x}, \mathrm{y}, \mathrm{y}) \mathrm{Be}$ Linearized ? Proceedings of Institute of Mathematics of NAS of Ukraine, 43(2), 27-35.

Karasu, A., \& Leach, P. G. L. (2009). Nonlocal symmetries and integrable ordinary differential equations: $\ddot{\mathrm{x}}+3 \times \dot{\mathrm{x}}+$ $\mathrm{x}^{3}=0$ and its generalizations. Journal of Mathematical Physics, 50(7), 0-17. https://doi.org/10.1063/1.3158856

Mahomed, F. M. (2007). Symmetry group classification of ordinary differential equations: Survey of some results. Mathematical Methods in the Applied Sciences, 30(16), 1995-2012. https://doi.org/10.1002/mma.934

Mustafa, M. T., Al-Dweik, A. Y., \& Mara'beh, R. A. (2013). On the linearization of second-order ordinary differential equations to the Laguerre form via generalized Sundman transformations. Symmetry, Integrability, and Geometry: Methods and Applications (SIGMA), 9(June 2014), 1-10. https://doi.org/10.3842/SIGMA.2013.041

Nakpim, W., \& Meleshko, S. V. (2010). Linearization of second-order ordinary differential equations by generalized Sundman transformations. Symmetry, Integrability, and Geometry: Methods and Applications (SIGMA), 6, 1-11. https://doi.org/10.3842/SIGMA.2010.051

Orverem, J. M., Azuaba, E., \& Balami., H. M. (2017). Linearization of the Equation of Motion of a Free Particle in a Space of Constant Curvature through Differential Forms. BAOBAB Journal of Science and Technology, 1(1), $116-122$.

Orverem, J. M., Haruna, Y., Abdulhamid, B. M., \& Adamu, M. Y. (2021). Linearization of Emden Differential Equation via the Generalized Sundman Transformations. Advances in Pure Mathematics, 11, $163-168$. https://doi.org/10.4236/apm.2021.113011

Orverem, J. M., Tyokyaa, R. K., \& Balami, H. M. (2017). Differential Forms : A Tool for Linearizing Second Order Ordinary Differential Equations. Journal of Scientific and Engineering Research, 4(10), 213-219.

\section{Copyrights}

Copyright for this article is retained by the author(s), with first publication rights granted to the journal.

This is an open-access article distributed under the terms and conditions of the Creative Commons Attribution license (http://creativecommons.org/licenses/by/4.0/). 